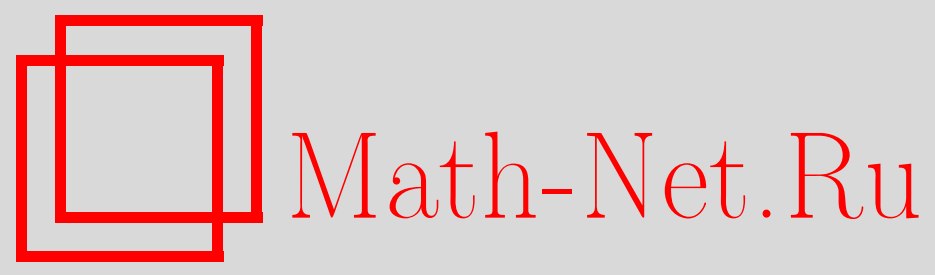

В. В. Терауд, Моделирование высокотемпературной осадки цилиндров при различных уравнениях ползучести материала, Вестн. Сам. гос. техн. ун-та. Сер. Физ.-мат. науки, 2011, выпуск 1(), 283-290

DOI: https://doi.org/10.14498/vsgtu915

Использование Общероссийского математического портала Math-Net.Ru подразумевает, что вы прочитали и согласны с пользовательским соглашением http: //www.mathnet.ru/rus/agreement

Параметры загрузки:

IP: 54.198 .187 .58

26 апреля 2023 г., 05:28:50 
УДК 539.376, 621.735.32

\title{
МОДЕЛИРОВАНИЕ ВЫСОКОТЕМПЕРАТУРНОЙ ОСАДКИ ЦИЛИНДРОВ ПРИ РАЗЛИЧНЫХ УРАВНЕНИЯХ ПОЛЗУЧЕСТИ МАТЕРИАЛА
}

\section{B. B. Терауд}

НИИ механики МГУ им. М. В. Ломоносова, 119192, Москва, Мичуринский пр., 1.

E-mails: ldrnww@gmail.com, loko@imec.msu.ru

\begin{abstract}
Исследуется осадка кругового иилиндра жесткими плитами в условиях ползучести. Рассматриваются три различные модели материала. Допускается образование бочкообразной бормы в прочессе осаживания (решение строится на основе пакета LS-DYNA). Проведены вычисления всех основных параметров при двух программах нагружения: при постоянной скорости сближения оснований иилиндра и при постоянной сжимающей силе. Вычисления показали, что энергия, затрачиваемая на осаживание иилиндра в первом варианте осаживания, меньше, чем во втором (в конкретных прочессах осадки разность составляет $8-10 \%)$.
\end{abstract}

Ключевые слова: круговые иилиндры, модели материалов, осадка, ползучесть, бочкообразование.

Введение. Технологические процессы обработки металлов обычно реализуются при комнатной температуре, и при расчётах этих процессов, как правило, используются модели, учитывающие только пластические свойства материала. В данном направлении известны классические результаты многих исследователей. В последние годы важные результаты получены С. Е. Александровым, А. Л. Воронцовым, И. В. Добровым и другими учеными.

При необходимости уменьшения сопротивления металлов необратимому деформированию технологические процессы следует проводить в условиях горячей обработки металлов. Кроме того, при проведении технологических процессов в условиях высоких температур уровень накапливаемых повреждений в металле значительно меньше, чем при комнатной температуре. При этих температурах, несмотря на сравнительно небольшое время деформирования, существенное значение имеет вязкость материала, и поэтому расчёты процессов обработки металлов следует проводить на основе уравнений теории ползучести. Технологические задачи в условиях ползучести исследовались Н. Н. Малининым, О. В. Сосниным, С. П. Яковлевым, С. С. Яковлевым, К.И. Романовым, В.П. Радченко и другими учёными. Следует отметить большой вклад Н. Н. Малинина [1] в решение различных задач (осадка, штамповка, прокатка, волочение и др.).

1. Постановка задачи. Рассматривается задача об осесимметричной осадке кругового цилиндра высотой $2 H_{0}$ и радиусом $R_{0}$ (рис. 1 ) между двумя абсолютно жесткими плитами с кинематической и силовой программами нагружения. В первом случае осаживание осуществляется сближением плит с не зависящей от времени $t$ скоростью $2 w(t)=2 w_{0}$, во втором - посредством

Валентин Викторович Терауд, младший научный сотрудник, лаб. ползучести и длительной прочности. 


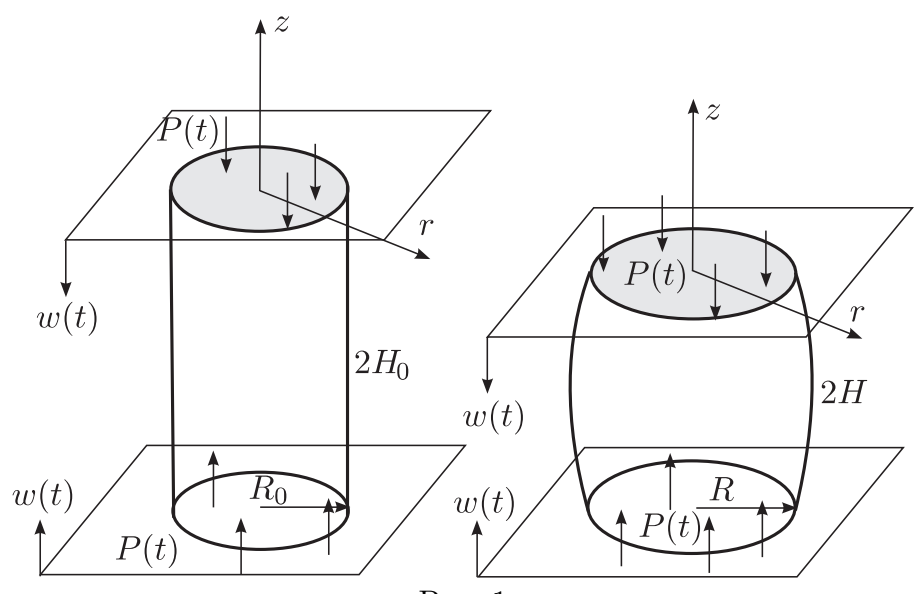

Рис. 1

постоянной сжимающей силы $P(t)=P_{0}$, приложенной к плитам. При этом учитываются только упругие деформации и деформации ползучести $p_{i j}$.

В качестве связи интенсивности напряжений $\sigma_{u}$ и интенсивностей деформаций ползучести $p_{u}$ или скоростей деформаций ползучести $\dot{p}_{u}$ принимается одна из трёх следующих моделей:

- степенная связь времени $t$ и интенсивностей напряжений и деформаций ползучести

$$
p_{u}=A \sigma_{u}^{n} t^{l} ;
$$

- степенная связь интенсивностей напряжений и скоростей деформаций ползучести

$$
\dot{p}_{u}=B \sigma_{u}^{m} ;
$$

- зависимость интенсивности скоростей деформации ползучести от интенсивностей напряжений и деформаций ползучести

$$
\dot{p}_{u} p_{u}^{\alpha}=C\left(\frac{\sigma_{u}}{\sigma_{b}-\sigma_{u}}\right)^{q}
$$

где $\sigma_{b}$ - предел кратковременной прочности материала при рассматриваемой температуре.

Решение задачи на основе соотношения (2) приведено в [2]. Условия на границах цилиндра в данной работе выбраны согласно закону Кулона с постоянным коэффициентом трения $\mu$ или законом Зейделя с полным контактным прилипанием. Решение задачи, основанное на предположении независимости всех характеристик напряжённо-деформируемого состояния от продольной координаты цилиндра, приведено в [3,4].

Разработанный в настоящей статье алгоритм решения задачи с учётом бочкообразования в сочетании с методом градиентного спуска обеспечивает эффективное решение значительно более широкого класса задач ползучести, чем алгоритм, приведенный в [2]. Основное внимание в данной работе уделено влиянию различных моделей, свойств материала, способа нагружения и других факторов на характеристики напряжённо-деформированного состояния 
цилиндра и на величину энергии, затрачиваемой на его осаживание. В качестве конкретного материала был выбран алюминиевый сплав Д16Т при температуре $400^{\circ} \mathrm{C}$.

2. Решение задачи. Моделирование процесса осадки цилиндра в рассматриваемых условиях проведено методом конечных элементов (MKЭ) с использованием программного кода LS-DYNA (LS-DYNA Theory 2006 (manual) http://www.lstc.com). При учёте условий симметрии в работе рассматривалась $1 / 8$ часть цилиндра, ограниченная плоскими сечениями $S_{1}(z=0)$ и $S_{2}$ $(\theta=0$ и $\theta=\pi / 2)$, координата $z=0$ соответствует центральному поперечному сечению цилиндра.

В прямоугольной декартовой системе координат $x_{1}=x, x_{2}=y, x_{3}=z$ положение произвольной точки цилиндра $x_{i}$ в момент времени $t$ с начальными координатами $\left(X_{1}, X_{2}, X_{3}\right)$ описывалось функциями $x_{i}\left(X_{\gamma}, t\right)(i=1,2,3$, $\gamma=1,2,3)$, где $x_{i}\left(X_{\gamma}, 0\right)=X_{\gamma}$. На сечениях $\theta=0, \theta=\pi / 2$ и $z=0$ учитывались условия симметрии.

Уравнения равновесия внутри цилиндра удовлетворялись в ослабленной формулировке в соответствии с принципом равенства виртуальных работ внутренних и внешних сил. Закон Кулона на контактной границе $S_{3}(z=H)$ между плитой и цилиндром задавался в следующем виде:

$$
\left.\sigma_{33}\right|_{S_{3}}=-g,\left.\quad \sigma_{12}\right|_{S_{3}}=\left.\sigma_{23}\right|_{S_{3}}=\mu g,
$$

где $g$ - абсолютная величина внешнего давления жестких плит на торцы цилиндра, $\mu$ - коэффициент трения.

В квазистатическом приближении при отсутствии массовых сил и с учётом (4) равенство виртуальных работ приводит к тождеству

$$
\int_{W} \sigma_{i j} \frac{\partial\left(\delta x_{i}\right)}{\partial x_{j}} d v+g \int_{S_{3}}\left(\delta x_{3}-\mu\left(\delta x_{1}+\delta x_{2}\right)\right) d s=0,
$$

здесь $W$ - объём рассматриваемой области цилиндра, $d v$ - элемент этого объёма, $d s-$-элемент контактной поверхности $S_{3}$.

При решении используется гипотеза пропорциональности девиаторов напряжений $s_{i j}$ и скоростей деформаций ползучести $\dot{p}_{i j}$ :

$$
\dot{s}_{i j}=2 G \dot{p}_{i j} \quad \text { при } \sigma_{u} \leqslant \sigma^{*}, \quad \frac{\dot{s}_{i j}}{\sigma_{u}}=\frac{2}{3} \frac{\dot{p}_{i j}}{\dot{p}_{u}} \quad \text { при } \sigma_{u}>\sigma^{*},
$$

где $\sigma^{*}$ - минимальное напряжение (предел ползучести), при котором в материале возникают деформации ползучести, $G$ - коэффициент, характеризующий жесткость материала в упругой зоне. При этом в качестве зависимости $\sigma_{u}$ от $p_{u}$ и $\dot{p}_{u}$ рассматривается одна из моделей:

$$
\begin{gathered}
\sigma_{u}=A^{-1 / n}\left(p_{u} \cdot t^{-l}\right)^{1 / n} \quad \text { для модели }(1), \\
\sigma_{u}=B^{-1 / m}\left(\dot{p}_{u}\right)^{1 / m} \quad \text { для модели }(2), \\
\sigma_{u}=\sigma_{b} \frac{M}{1+M}, \quad M=\left(\frac{p_{u}^{\alpha} \dot{p}_{u}}{C}\right)^{1 / q} \text { для модели }(3) .
\end{gathered}
$$


Связь между гидростатическим давлением $\sigma_{0}$ и объёмной деформацией $\varepsilon_{0}$ полагается линейной: $\sigma_{0}=3 K \varepsilon_{0}$, где $K$ - модуль объёмного сжатия.

Программа для моделей (8), (9) была написана на языке Fortran и интегрировалась в конечно-элементный комплекс LS-DYNA средствами пользовательского программирования. Дискретизация по пространственным координатам $x_{i}=(x, y, z)$ осуществлялась посредством 8-узловых гексаэдрических элементов с функциями формы

$$
\phi_{j}=\frac{1}{8}\left(1+\xi \xi_{j}\right)\left(1+\eta \eta_{j}\right)\left(1+\zeta \zeta_{j}\right)
$$

так что

$$
x_{i}\left(X_{\alpha}, t\right)=x_{i}\left(X_{\alpha}(\xi, \eta, \zeta), t\right)=\sum \phi_{j}(\xi, \eta, \zeta) x_{i}^{j}(t),
$$

$x_{i}^{j}$ - координата узла с номером $j$ в направлении $i$, а $\left(\xi_{j}, \eta_{j}, \zeta_{j}\right)$ соответствовали 8 вариантам упорядоченных троек со значениями $( \pm 1)$ для соответствующих узлов гексаэдра: $(-1,-1,-1)$ для $j=1 ;(1,-1,-1)$ для $j=2 ;(1,1,-1)$ для $j=3$ и так далее до $j=8$. Были введены обозначения

$$
\begin{aligned}
& N(3 \times 24)=\left[\begin{array}{llllllllll}
\phi_{1} & & & \phi_{2} & & & \ldots & \phi_{8} & & \\
& \phi_{1} & & & \phi_{2} & & \ldots & & \phi_{8} & \\
& & \phi_{1} & & & \phi_{2} & \ldots & & & \phi_{8}
\end{array}\right] \text {, } \\
& \sigma=\left(\sigma_{11}, \sigma_{22}, \sigma_{33}, \sigma_{12}, \sigma_{23}, \sigma_{31}\right)^{\top}, \quad T=(-g, \mu g, \mu g)^{\top}, \\
& B=\left[\begin{array}{ccc}
\frac{\partial}{\partial x} & 0 & 0 \\
0 & \frac{\partial}{\partial y} & 0 \\
0 & 0 & \frac{\partial}{\partial z} \\
\frac{\partial}{\partial y} & \frac{\partial}{\partial x} & 0 \\
0 & \frac{\partial}{\partial z} & \frac{\partial}{\partial y} \\
\frac{\partial}{\partial z} & 0 & \frac{\partial}{\partial x}
\end{array}\right] N
\end{aligned}
$$

с учётом которых, а также уравнения (10), принцип равенства виртуальных работ (5) привёлся к виду

$$
\sum_{m=1}^{f}\left\{\int_{W_{m}} B^{\top} \sigma d v-\int_{S_{3 m}} N^{\top} T d s\right\}=0
$$

где $f$-количество элементов в конечно-элементной модели. Соотношение (11) можно переписать в матричной форме:

$$
F_{1}(\dot{X}, X, t)-F_{2}(X, t)=0
$$

где $X^{\top}=\left(x_{i}^{j}\right)_{j=1}^{l}$ - искомый вектор-столбец узловых координат.

Дискретизация по времени выполнялась по неявной схеме. Нелинейное уравнение (12) решалось модифицированным методом Ньютона-Рафсона с постоянным секущим модулем, равным начальному модулю упругости $G$. 
Для перерасчёта поля напряжений на новом временном слое $(k+1)$ в соответствии с гипотезой о пропорциональности девиаторов напряжений и скоростей деформаций ползучести (6) и одной из связей интенсивностей напряжений и скоростей деформаций (7)-(9) использовался метод радиального спуска. Предполагалось, что реализуется деформация по траектории малой кривизны, согласно этому вначале выполнялся чисто упругий расчёт с заданными значениями приращений деформаций:

$$
\begin{gathered}
\widehat{s}_{i i}^{k+1}=\left(\sigma_{i i}^{k}-\sigma_{0}^{k}\right)+2 G\left(\Delta \varepsilon_{i i}^{k+1}-\Delta \varepsilon_{0}^{k+1}\right) \quad(i=1,2,3), \\
\widehat{s}_{i j}^{k+1}=\sigma_{i j}^{k}+G \Delta \varepsilon_{i j}^{k+1} \quad(i \neq j, \quad i=1,2,3, j=1,2,3), \\
\widehat{\sigma}_{u}^{k+1}=\sqrt{\frac{3}{2} \sum\left(\widehat{s}_{i j}^{k+1}\right)^{2}}, \quad p_{u}^{k+1}=\frac{1}{\Delta t} \cdot \sqrt{\frac{2}{3} \sum\left(\Delta \varepsilon_{i j}^{k}\right)^{2}}, \quad \Delta t=t^{k+1}-t^{k} .
\end{gathered}
$$

Затем компоненты девиатора тензора напряжений $\widehat{\sigma}_{u}^{k+1}$ масштабировались по выбранной модели из (7)-(9):

$$
\bar{\sigma}_{u}^{k+1}=\left[\begin{array}{ll}
A^{-1 / n}\left(p_{u}^{k+1} \cdot\left(t^{k+1}\right)^{-l}\right)^{1 / n} & \text { для модели (1), } \\
B^{-1 / m}\left(\dot{p}_{u}^{k+1}\right)^{1 / m} & \text { для модели (2), } \\
\sigma_{b} M /(1+M), M=C^{-1 / q}\left(\dot{p}_{u}^{k+1}\right)^{1 / q}\left(p_{u}^{k+1}\right)^{\alpha / q} & \text { для модели (3), } \\
s_{i j}^{k+1}=\widehat{s}_{i j}^{k+1} \cdot \frac{\bar{\sigma}_{u}^{k+1}}{\widehat{\sigma}_{u}^{k+1}} . &
\end{array}\right.
$$

3. Результаты вычислений. Материальные константы для (1)-(3) выбирались в соответствии со свойствами сплава Д16Т при температуре $400{ }^{\circ} \mathrm{C}$. Мeтодика по определению материальных констант для (2) подробно изложена в [5]. Для модели (3) коэффициенты $C$ и $q$ выбирались таким образом, чтобы характеристики ползучести сплава Д16Т в диапазоне напряжений 50-80 МПа аппроксимировались с наименьшей погрешностью. Такой выбор обусловлен тем, что при осадке цилиндра характерное напряжение $\sigma_{z}$ находится именно в этом диапазоне. Материальные константы для моделей (1), (2) определялись с помощью экспериментальных данных в диапазоне 20-80 МПа. Полученные материальные константы для (1)-(3) представлены в таблице.

\begin{tabular}{c|c|c||c|c||c|c|c|c}
\hline$n$ & $l$ & $A, \mathrm{M \Pi а}^{-n} \cdot \mathrm{ceK}^{-l}$ & $m$ & $B, \mathrm{M \Pi a}^{-m} \cdot \mathrm{ceK}^{-1}$ & $\alpha$ & $q$ & $\sigma_{b}, \mathrm{M \Pi а}$ & $C, \mathrm{ceK}^{-1}$ \\
\hline 11,1 & 1 & $3,29 \cdot 10^{-22}$ & 11,1 & $3,29 \cdot 10^{-22}$ & 0 & 2,3 & 88,1 & $1,2 \cdot 10^{-3}$ \\
\hline
\end{tabular}

В качестве модельного примера рассматривался цилиндр с радиусом $R_{0}=$ $=19,5$ мм и высотой $2 H_{0}=39$ мм. При моделировании различных программ нагружения были выбраны следующие параметры осадки: $w_{0}=0,013$ мм $/ \mathrm{c}$, $P_{0}=73 \mathrm{kH}$.

На рис. 2 приведены расчётные зависимости высоты цилиндра от времени полученные по моделям (1)-(3) при его осаживании по силовой программе $P(t)=P_{0}$. Значения $R_{0}, H_{0}, P_{0}$ и $t$ для приведённых расчётов выбирались одинаковыми. Цифры на рис. 2 соответствуют номеру модели. Модели материала (1) и (2) дают схожие кривые (немного различающиеся в середине процесса осадки и совпадающие в конце осадки). Модель (3) приводит 
к несколько большей осадке за то же время. В [5] автором приведены результаты экспериментального исследования, из которых следует, что модель (3) приводит к наиболее близкому соответствию с экспериментальными данными.

На рис. 3 приведены зависимости высоты цилиндра $2 H$ от времени $t$ для различных значений коэффициента трения по программе осадки $P(t)=$ const для модели (3). Цифрами обозначены кривые при следующих условиях: 1) $\mu=$ $=0$; 2$) \mu=0,5$; 3) $\mu=\mu^{*}$, коэффициент трения $\mu^{*}$ соответствует прилипанию материала на контактной плоскости (далее кратко - прилипание). Полное контактное прилипание можно рассматривать как следствие максимально достижимого коэффициента трения $\mu^{*}$. Из данных, представленных на рис. 3 , следует, что при прочих равных параметрах с увеличением коэффициента трения высота цилиндра в течение 740 сек уменьшается на $20 \%$.

На рис. 4 и 5 приведены зависимости $P(t), w(t)$ и энергии деформирования $V(t)$ для двух $(P(t)=$ const и $w(t)=$ const $)$ рассмотренных программ нагружения при $\mu=0$ (соответственно линии 1,2$)$ и $\mu=\mu^{*}$ (соответственно линии $3,4)$. Для обеспечения одинаковой высоты цилиндра в обеих программах нагружения при осаживании по программе $P(t)=$ const сила осаживания для нулевого трения была взята равной $74 \mathrm{kH}$, а для случая прилипания $-87 \mathrm{kH}$. Подобные параметры обеспечили конечную высоту цилиндра при $t=740$ сек на уровне 29,5 мм в обеих программах нагружения и при обоих значениях коэффициентах трения $\left(\mu=0\right.$ и $\left.\mu=\mu^{*}\right)$.

На рис. 4 представлены зависимости сжимающей силы $P(t)$ и скорости осадки $w(t)$. Из данных, представленных на рис. 4, следует, что зависимость $P(t)$ при постоянном значении $w_{0}$ является возрастающей функцией времени, причём для $\mu=\mu^{*}$ значение $P(t)$ на $30 \%$ больше, чем для значения $\mu=0$.

На рис. 5 представлены зависимости энергии $V(t)$, затрачиваемой на оса-
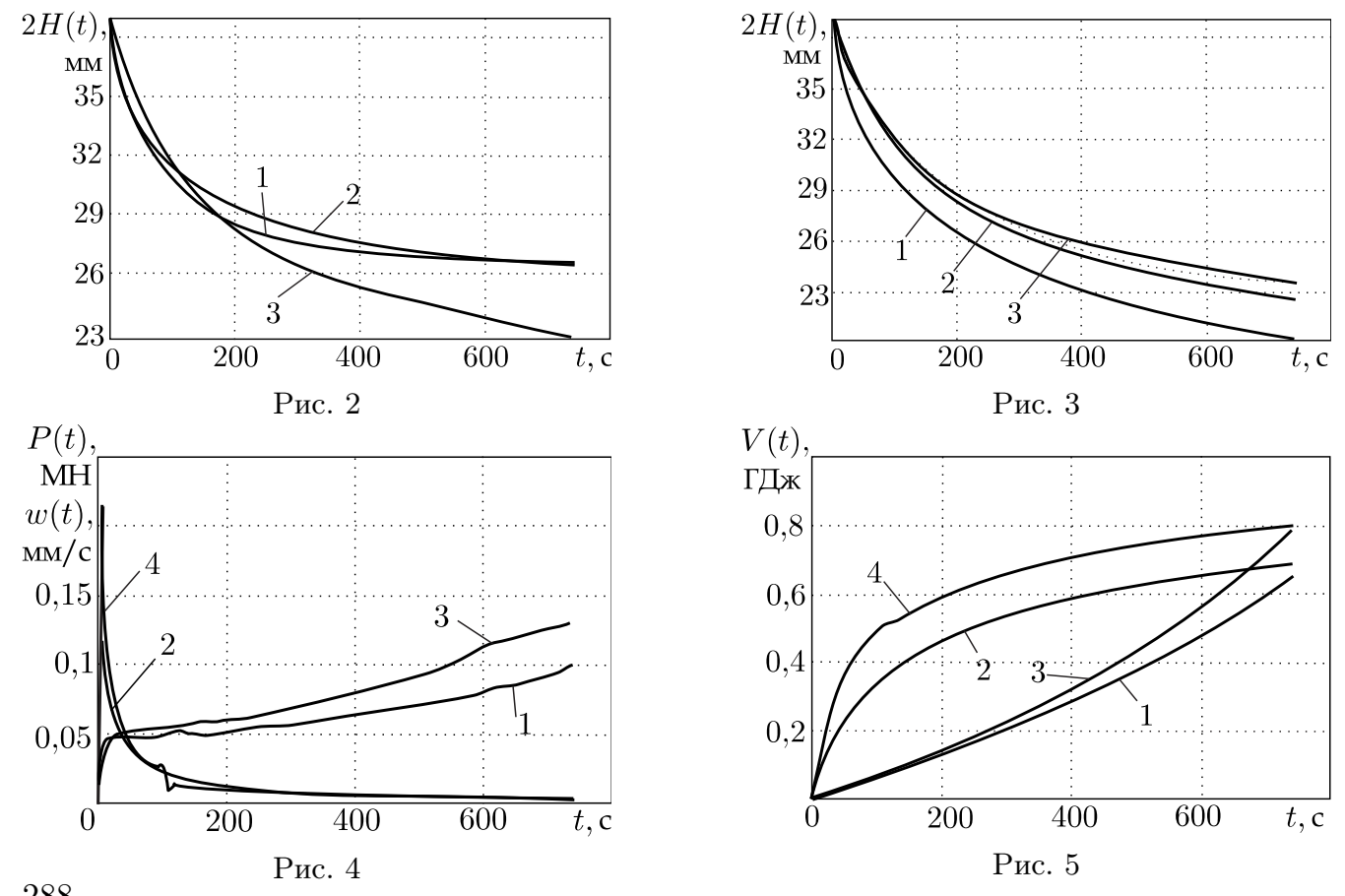
живание цилиндра, от времени $t$. Представляет интерес сравнение значений $V$ для различных программ нагружения. Анализ показывает, что при любых моделях материала энергия деформирования $V_{w}$, затрачиваемая на осадку цилиндра при постоянной скорости сближения плит (кривые 1 и 3 соответственно), меньше, чем энергия деформирования $V_{P}$, накопленная при постоянной сжимающей силе (кривые 2 и 4). Вычисления показали, что разность $\left(V_{P}-V_{w}\right)$ возрастает с увеличением коэффициента трения $\mu$ и отношения $R_{0} / H_{0}$

На рис. 6 приведена зависимость бочкообразности

$$
B(t)=\left(D_{\max }(t)-D_{\min }(t)\right) / D_{\max }(t)
$$

от типа нагружения и контактного трения. Здесь линии 1, 2 получены по кинематической программе нагружении при коэффициентах трения $\mu=0,5$ и $\mu=\mu^{*}$ соответственно, а линии 3,4 - по силовой программе нагружения с теми же значениями коэффициентов трения. Как видно из данных, представленных на рис. 6 , наибольшее бочкообразование происходит при силовом способе осаживания при контактном прилипании. Наименьшую бочкообразность цилиндр в процессе осаживания сохраняет при небольших коэффициентах трения и кинематическом способе осаживания.

На рис. 7 приведены зависимости продольной поверхностной логарифмической деформации $\bar{p}_{z}(t)$ элемента цилиндра в его середине при различных коэффициентах трения. Здесь линия 1 получена при $\mu=0$, линия 2 -при $\mu=0,5$; линия $3-$ при $\mu=\mu^{*}$. Следует учесть, что величина $\bar{p}_{z}$ отличается от $p_{z}$ всего цилиндра, так как часть боковой поверхности переходит на контактную плоскость вблизи сжимающих плит, в связи с этим $\bar{p}_{z}<p_{z}$. Из данных, представленных на рис. 7 , следует, что с увеличением коэффициента трения поверхностная деформация уменьшается. Это явление связано с тем, что материал вблизи контакта активно переходит на торцевую поверхность, препятствуя деформированию материала в центральной части цилиндра.
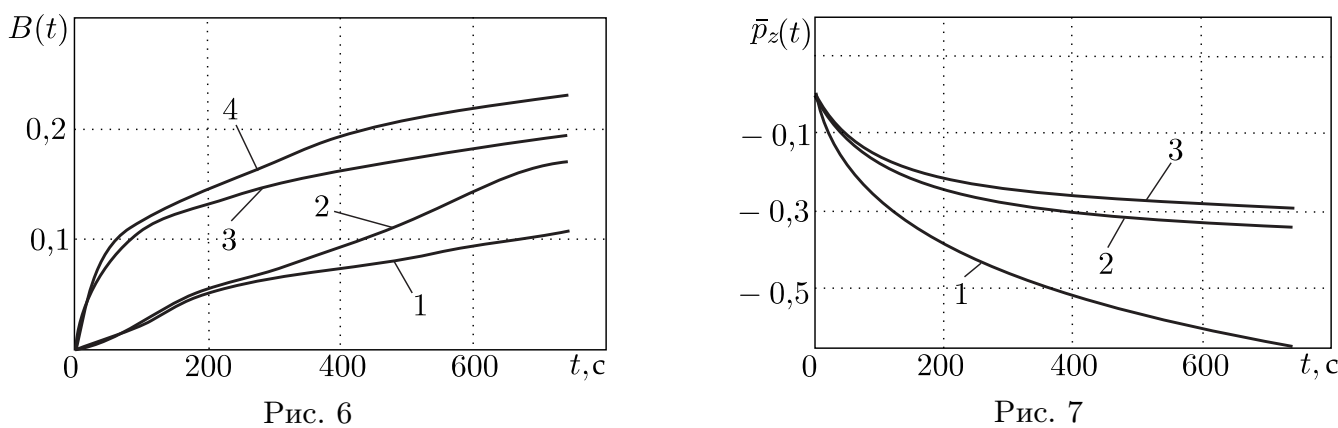

Автор выражает благодарность А. М. Локощенко за постановку задачи и консультации и П. А. Моссаковскому за оказанную помощь при проведении вычислений.

Работа выполнена при поддержке РФФИ (проект № 11-08-00007). 


\section{БИБЛИОГРАФИЧЕСКИЙ СПИСОК}

1. Малинин Н. Н. Ползучесть в обработке металлов. М.: Машиностроение, 1986. 216 с. [Malinin N. N. Creep in metal forming. Moscow: Mashinostroenie, 1986. 216 pp.]

2. Локощенко А.М., Моссаковский П. А., Терауд В. В. Исследование осадки круговых цилиндров при ползучести с учетом и без учета бочкообразования // Вычислителъная механика сплошных сред, 2010. Т. 3, № 1. C. 52-62. [Lokoshchenko A. M., Mossakovsky P. A., Teraud $V$. $V$. Issledovanie osadki krugovyh tsilindrov pri polzuchesti s uchetom i bez ucheta bochkoobrazovaniya // Vychislitel'naya mehanika sploshnyh sred, 2010. Vol. 3, no.1. Pp. 52$62]$.

3. Локощенко A.М., Демин В.А., Носов В.В. Осадка кругового цилиндра в условиях установившейся ползучести // Изв. вуз. Машиностроение, 2007. №4. С. 3-10. [Lokoshchenko A.M., Demin V.A., Nosov V.V. Osadka krugovogo tsilindra v usloviyah ustanovivsheysya polzuchesti // Izv. vuz. Mashinostroenie, 2007. no. 4. Pp. 3-10].

4. Локощенко A. М. Моделирование процесса ползучести и длительной прочности металлов. М.: МГИУ, 2007. 264 с. [Lokoshchenko A. M. Simulation of the creep and long-term strength of metals. Moscow: MGIU, 2007. 264 pp.]

5. Терауд В.В. Инновационная многокомпонентная система бесконтактных измерений при высокотемпературных испытаниях / В сб.: Тр. конф.-конкурса молодых ученых Института механики МГУ (14-16.10.2009). М.: МГУ, 2010. С. 307-317. [Teraud $V . V$. Innovatsionnaya mnogokomponentnaya sistema beskontaktnyh izmereniy pri vysokotemperaturnyh ispytaniyah/In: Tr. konf.-konkursa molodyh uchenyh Instituta mehaniki MGU. Moscow: MGU, 2010. Pp. 307-317].

Поступила в редакцию 20/VII/2010;

в окончательном варианте - 18/II/2011.

MSC: 74D10; 74S05

\section{SIMULATION OF HIGH-TEMPERATURE SHORTENING OF CYLINDERS UNDER DIFFERENT MATERIAL CREEP EQUATIONS}

\section{V.V. Teraud}

Institute of Mechanics, Lomonosov Moscow State University,

1, Michurinskiy pr., Moscow, 119192, Russia.

E-mails: ldrnww@gmail.com, loko@imec.msu.ru

A subsidence of circular cylinders between rigid stamps at creep conditions is studied. There are three different material models. Appearance of barrel shape form is allowed (this decision is realized on the basis of package LS-DYNA). Calculations of all principal parameters were carried out at two loading programs: at constant speed of the cylinders' bases coming together and at constant compressing force. Calculations have shown that the energy, spent on subsidence of the cylinder during the first loading variant isless, than in the second variant (this difference in certain processes is equal to $8-10 \%)$.

Key words: circular cylinders, material models, shortening, creep, barrel shape form.

Original article submitted 20/VII/2010; revision submitted 18/II/2011.

Valentin V. Teraud, Junior Researcher, Lab. of Creep \& Long-Term Strength. 